\title{
Deep Searches for High Redshift Molecular Absorption
}

\author{
S. J. Curran and J. K. Webb
}

School of Physics, University of New South Wales, NSW 2052, Australia

\author{
M. T. Murphy \\ Institute of Astronomy, Madingley Road, Cambridge CB3 OHA, UK \\ N. Kuno \\ Nobeyama Radio Observatory, Nagano 384-1305, Japan
}

\begin{abstract}
Millimetre-band scans of the frequency space towards optically dim quasars is potentially a highly efficient method for detecting new high redshift molecular absorption systems. Here we describe scans towards 7 quasars over wide bandwidths (up to $23 \mathrm{GHz}$ ) with sensitivity limits sufficient to detect the 4 redshifted absorbers already known. With wider frequency bands, highly efficient searches of large numbers of possibly obscured objects will yield many new molecular absorbers.
\end{abstract}

\section{Introduction}

Webb et al. (these proceedings) discussed constraints on possible variations in fundamental constants offered by quasar absorption lines. Optical studies (Webb et al. 1999; Murphy et al. 2003) find a statistically significant variation of the fine-structure constant, $\Delta \alpha / \alpha \approx(-0.54 \pm 0.12) \times 10^{-5}$, over the redshift range $0.2<z_{\text {abs }}<3.7$. Comparison between $\mathrm{H} \mathrm{I}-21 \mathrm{~cm}$ and molecular rotational (millimetre) absorption lines can yield an order of magnitude better precision (per absorption system) than these purely optical constraints (Drinkwater et al. 1998; Carilli et al. 2000; Murphy et al. 2001): a statistical sample of $\mathrm{H} \mathrm{I}-21 \mathrm{~cm} / \mathrm{mm}$ comparisons will provide an important cross-check on varying- $\alpha$. Currently, however, only 4 such redshifted millimetre absorption systems are known (Wiklind, these proceedings). To increase this number we have employed the following search strategies:

1. Deep integrations of damped Lyman-alpha absorbers (DLAs), the highest column density $\left(N_{\mathrm{HI}} \gtrsim 10^{20} \mathrm{~cm}^{-2}\right)$ quasar absorbers known. Since we observe at a known redshift and therefore frequency, optical depth limits better than $\tau \lesssim 0.1$ are often obtained. The DLA results are discussed in detail by Curran et al. (2004).

2. Scanning the frequency space toward visually dim millimetre bright quasars in search of a possible absorber responsible for the visual obscuration. Here we summarize our results as obtained with the Swedish-ESO Sub- 
millimetre Telescope (SEST) and Nobeyama Radio Observatory's 45-m telescope (NRO).

\section{Results}

From an extensive literature search we selected four millimetre-loud quasars yet to be optically identified (Table 1, top). For each of these we performed a

Table 1. The SEST (top) and NRO (bottom) search results. $V$ is the visual magnitude with the Galactic extinction, $A_{B}$, given. $z_{\mathrm{em}}$ is the quasar redshift, $S$ the approximate flux density in Jy at the observed frequency band, $\nu$, and $\tau$ is the typical $3 \sigma$ optical depth limit (quoted for a resolution of $1 \mathrm{~km} \mathrm{~s}^{-1}$, see Curran et al. 2002).

\begin{tabular}{lccccccc}
\hline Quasar & $V$ & $A_{B}$ & $z_{\mathrm{em}}$ & Ref & $S$ & $\nu[\mathrm{GHz}]$ & $\tau$ \\
\hline $0500+019$ & 21.2 & 0.289 & 0.58457 & 2 & 0.5 & $78.30-80.90$ & 2 \\
$\ldots$ & $\ldots$ & $\ldots$ & $\ldots$ & $\ldots$ & 0.5 & $85.50-86.50$ & 0.8 \\
$\ldots$ & $\ldots$ & $\ldots$ & $\ldots$ & $\ldots$ & 0.5 & $112.10-113.10$ & 0.8 \\
$\ldots$ & $\ldots$ & $\ldots$ & $\ldots$ & $\ldots$ & 0.4 & $130.00-141.40$ & 1 \\
$0648-165$ & - & 2.456 & - & - & 1.6 & $78.30-80.90$ & 0.2 \\
$\ldots$ & $\ldots$ & $\ldots$ & $\ldots$ & $\ldots$ & 1.5 & $83.90-90.50$ & 0.2 \\
$\ldots$ & $\ldots$ & $\ldots$ & $\ldots$ & $\ldots$ & 0.7 & $138.00-141.40$ & 0.5 \\
$\ldots$ & $\ldots$ & $\ldots$ & $\ldots$ & $\ldots$ & 0.6 & $142.80-149.40$ & 0.7 \\
$0727-115$ & 22.5 & 1.271 & - & - & 2.9 & $78.30-80.90$ & 0.2 \\
$\ldots$ & $\ldots$ & $\ldots$ & $\ldots$ & $\ldots$ & 2.7 & $83.90-90.50$ & 0.1 \\
$\ldots$ & $\ldots$ & $\ldots$ & $\ldots$ & $\ldots$ & 1.5 & $138.00-141.40$ & 0.2 \\
$\ldots$ & $\ldots$ & $\ldots$ & $\ldots$ & $\ldots$ & 1.2 & $142.80-148.60$ & 0.4 \\
$1213-172$ & 21.4 & 0.253 & - & - & 1.0 & $78.30-80.90$ & 0.4 \\
$\ldots$ & $\ldots$ & $\ldots$ & $\ldots$ & $\ldots$ & 0.9 & $83.90-90.50$ & 0.4 \\
$\ldots$ & $\ldots$ & $\ldots$ & $\ldots$ & $\ldots$ & 0.5 & $138.00-141.40$ & 0.8 \\
$\ldots$ & $\ldots$ & $\ldots$ & $\ldots$ & $\ldots$ & 0.4 & $142.80-151.00$ & 1 \\
\hline $0742+103$ & $\sim 24$ & 0.111 & - & - & 0.6 & $46.90-47.50$ & - \\
$\ldots$ & $\ldots$ & $\ldots$ & $\ldots$ & $\ldots$ & 0.4 & $77.25-87.50$ & 0.9 \\
$\ldots$ & $\ldots$ & $\ldots$ & $\ldots$ & $\ldots$ & 0.4 & $88.45-89.35$ & 0.4 \\
$1600+335$ & 23.2 & 0.137 & 1.1 & 3 & 0.7 & $46.90-47.50$ & 2 \\
$\ldots$ & $\ldots$ & $\ldots$ & $\ldots$ & $\ldots$ & 0.5 & $77.25-87.50$ & 0.5 \\
$\ldots$ & $\ldots$ & $\ldots$ & $\ldots$ & $\ldots$ & 0.5 & $88.45-89.35$ & 0.4 \\
$1655+077$ & 20.1 & 0.66 & 0.621 & 1 & 1.5 & $46.90-47.50$ & 1 \\
$\ldots$ & $\ldots$ & $\ldots$ & $\ldots$ & $\ldots$ & 1.2 & $77.25-87.50$ & 0.7 \\
$\ldots$ & $\ldots$ & $\ldots$ & $\ldots$ & $\ldots$ & 1.2 & $88.45-89.35$ & 0.3 \\
\hline
\end{tabular}

References: (1) Wilkes (1986), (2) Carilli et al. (1998), (3) Snellen et al. (2000).

spectral scan along the line-of-sight. The high sensitivity and large bandwidth ( $1 \mathrm{GHz}$ ), combined with the possibility of observing simultaneously with two receivers, permitted us to scan a range of $\approx 10 \mathrm{GHz}$ in both the 2-mm and 3 -mm bands. Over these ranges we reached optical depth limits in both bands 
sensitive enough the detect the 4 known redshifted millimetre absorbers (Table 1 cf. $\tau \approx 0.7$ to $\approx 2$ at $\gtrsim 4 \mathrm{~km} \mathrm{~s}^{-1}$ resolution), although no $\geq 3 \sigma$ absorption features were found (Murphy, Curran \& Webb 2003).

Of the remaining visually dim quasars, 10 have 3 - $\mathrm{mm}$ flux densities $\gtrsim 0.5$ Jy. Four of these are located in the north and with the $\mathrm{NRO}^{10}$ we were able to observe the three listed in Table 1 . While the $6-\mathrm{mm}$ limits are poor, again at 3 -mm our search is sensitive enough to detect the 4 known absorbers over the observed redshift range: For the $J=0 \rightarrow 1,1 \rightarrow 2$ and $2 \rightarrow 3$ of transitions $\mathrm{CO}, \mathrm{HCN}$ and $\mathrm{HCO}^{+}$, i.e. the most commonly detected transitions in the 4 known absorbers, the observed frequencies give a $50 \%$ coverage for $0742+103$ up to $z \approx 3$ and $30 \%$ for both $1600+335$ and $1655+077$ up to the emission redshift ${ }^{11}$. The coverage for the SEST sources are discussed in Murphy, Curran $\&$ Webb (2003); for the above transitions up to $90 \%$ is achieved due to the large bandwidth and dual receiver capability.

\section{References}

Carilli, C.L., Menten, K.M., Reid, M.J., Rupen, M.P., \& Yun, M.S. 1998, ApJ, 494, 175 Carilli, C.L., Menten, K.M., Stocke, J.T., Perlman, E., Vermeulen, R., Briggs, F., de Bruyn, A.G., Conway, J., \& Moore, C.P. 2000, PhRvL, 85, 5511

Curran, S.J., Murphy, M.T., Webb, J.K., Rantakyrö, F., Johansson, L. E.B., \& Nikolić, S. 2002, A\&A, 394, 763

Curran, S.J., Murphy, M.T., Pihlström, Y.M., Webb, J.K., Bolatto, A.D., \& Bower, G.C. 2004, MNRAS, 345, 609

Drinkwater, M.J., Webb, J.K., Barrow, J.D., \& Flambaum, V.V. 1998, MNR.AS, 295, 457

Murphy, M.T., Curran, S.J., \& Webb, J.K. 2003, MNRAS, 342, 830

Murphy, M.T., Webb, J.K., \& Flambaum, V.V. 2003, MNRAS, 345, 609

Murphy, M.T., Webb, J.K., Flambaum, V.V., Drinkwater, M.J., Combes, F., \& Wiklind, T. 2001, MNRAS, 327, 1244

Snellen, I.A.G., Schilizzi, R.T., Miley, G.K., de Bruyn, A.G., Bremer, M.N., \& Röttgering, H.J.A. 2000, MNRAS, 319, 445

Webb, J.K., Flambaum, V.V., Churchill, C.W., Drinkwater, M.J., \& Barrow, J.D. 1999, PhRvL, 82, 884

Wilkes, B.J. 1986, MNRAS, 218, 331

${ }^{10}$ The fact that each of the $6 \mathrm{AOSs}$ on the NRO only covers $0.25 \mathrm{GHz}$ is compensated by the high efficiency of the $45-\mathrm{m}$ antenna $\left(4 \mathrm{Jy} \mathrm{K}^{-1}\right.$ cf. $25 \mathrm{Jy} \mathrm{K}^{-1}$ at SEST).

${ }^{11}$ Note that we have included the possibility of $\mathrm{HCN}$ or $\mathrm{HCO}^{+} 0 \rightarrow 1$ Galactic absorption towards all 3 sources as well as $\mathrm{HCN}$ or $\mathrm{HCO}^{+} 1 \rightarrow 2$ in the host of $1600+335$. 